Investigaciones Feministas

ISSN-e: 2171-6080

http://dx.doi.org/infe.64110

\title{
Configuraciones subjetivas y discursivas de la vivencia materna: de maternidades "encarnadas" y activismos emancipatorios
}

\author{
Núria Calafell Sala ${ }^{1}$
}

Recibido: Abril 2019/ Revisado: Febrero 2020 / Aceptado: Marzo 2020

Resumen Este artículo aborda dos propuestas de la sociedad civil pertenecientes al Movimiento Activista Materno en Argentina, con el propósito de describir y analizar qué tipo de retóricas se ponen en juego en contextos de reapropiación subjetiva y discursiva de la vivencia materna, en especial en un momento de gran auge de los activismos de género en América Latina. Para ello, se utiliza la etnografía feminista como metodología de análisis que apunta al conocimiento (multi)situado, al reconocimiento de las madres como sujetas creadoras culturales, y a la identificación e interpretación de aquellos sesgos de género que aparecen en el lenguaje y que son reproducidos en los discursos. Como técnica, el Análisis Crítico del Discurso permite atender textualidades de diversa naturaleza semiótica (conversaciones de la realidad física y narrativas online) y desentrañar los elementos de poder y de desigualdad que se reproducen en ellas, a veces de manera implícita, otras veces explícitamente. Los resultados muestran que la maternidad sigue siendo un campo de disputas entre los procesos socioculturales que despliegan sobre ella una serie de tecnologías (entre ellas, las de género) y las reapropiaciones de sentido que llevan a cabo las subjetividades en su vivencia personal y colectiva.

Palabras clave: maternidad; vivencia de la maternidad; género; feminismos; doulas; parto respetado/humanizado.

[en] Subjective and discoursive configurations of mothering: embodied motherhoods and emancipatory activisms

\begin{abstract}
This article tackles two proposals of civil society belonging to Maternal Activist Movement in Argentina. The goal is to describe and analyse what kind of rhetoric are placed in contexts of both subjective and discursive re-appropriations of mothering, especially at a time of gender activism in Latin America. To that end, ethnography is used as a methodology of analysis that points to (multi)situated knowledge, to recognition of mothers as individual cultural creators, and to identification and interpretation of these gender biases that are included in language and are reproduced in discourses. As a tool, the Critical Analysis of Discourse allows to attend textualities of varying semiotic nature (conversations of physical reality and online narratives) and to clarify the elements of power and inequality that they replicate, sometimes implicitly, other times explicitly. The results show that motherhood continues to be a field of disputes between sociocultural processes that deploy on it some technologies (including gender technologies) and the re-appropriations of meanings carried out by subjectivities in their personal and collective experience.
\end{abstract}

Keywords: motherhood; mothering; gender; feminisms; doulas; respected/humanized childbirth.

Sumario. 1. Introducción. 2. Metodología. 3. Análisis. 3.1. Los encuentros de doulas: la maternidad encarnada. 3.2. Mujeres por un Parto Respetado Córdoba: activismos emancipatorios. 4. Conclusiones. Referencias bibliográficas.

Cómo citar: Calafell Sala, N. (2020). Configuraciones subjetivas y discursivas de la vivencia materna: de maternidades “encarnadas" y activismos emancipatorios, en Revista de Investigaciones Feministas 11(1), 101-111. 


\section{Introducción}

El lema con el que la Campaña Nacional por el Derecho al Aborto Legal, Seguro y Gratuito acompañó el último gran debate sobre este derecho reproductivo pendiente en Argentina, "La maternidad será deseada o no será" (2018) evidencia el carácter central que esta experiencia ocupa en los procesos de subjetivación, así como su necesaria relocalización dentro de las relaciones de poder que median lo económico, lo político y lo social (Sau, 2004: 21).

Como práctica cultural, la maternidad ha sido objeto de interpretaciones y escrituras diversas a lo largo del tiempo. Una de estas la concibió como un "bien común" (Federici, 2015: 173), cuyas posibilidades fisiológicas -gestar, parir, amamantar y criar- legitimaban el desplazamiento de la sujeto mujer de la esfera pública, y justificaban el control, la regulación e intervención sobre su cuerpo (Chodorow, 1984). Esto no solo favoreció que las mujeres fueran apartadas del mundo laboral y encerradas en el espacio doméstico, sino que, a medida que fueron incorporándose al ámbito público, las desigualdades se manifestaran tanto en el trabajo (Felitti, 2011a: 11) como en la casa (Federici, 2013). La célebre frase "Ellos dicen que se trata de amor. Nosotras que es trabajo no pago" (1975), recuperada en la actualidad en ciertos circuitos, condensaba en dos ideas fundamentales las dificultades que enfrentaban y siguen enfrentando diariamente quienes, por decisión propia o por mandato, se convierten en madres en nuestras sociedades contemporáneas. Por un lado, la reducción del amor materno a factores biológicos; y, por el otro, la desvalorización y consiguiente devaluación de la fuerza reproductiva y de las tareas de cuidado.

Ambas cuestiones han sido abordadas por el feminismo, siendo la primera de ellas la que más reflexiones ha suscitado. En especial, desde que Simone de Beauvoir (2005 [1949]) señalara el carácter construido de la experiencia materna y denunciara su centralidad para someter y reducir el deseo femenino a una única posibilidad: ser madre. Desde entonces, a pesar de que los abordajes con perspectiva de género han sido múltiples (Palomar 2005; Saletti, 2008) y varias han sido las voces "que" se han hecho eco de la ambivalencia y la inconsistencia del deseo materno (Quinzio, 1999; Rich, 2019 [1976]), la tendencia en general dentro del pensamiento feminista ha sido la de denunciar la maternidad como una forma más de opresión del patriarcado.

Una explicación plausible ante esta predominancia se encuentra en los modos representacionales que configuran un modelo de maternidad unívoco, abstracto, despolitizado, des-sexualizado y des-socializado, en el decir de Brigitte Vasallo (2014). En un artículo reciente, Mariona Visa explica cómo, desde finales de los años ochenta del siglo pasado, la figuración de la maternidad en medios masivos de comunicación como la televisión se construyó sobre la categoría posteriormente denominada "maternidad intensiva" (Hays, 1998). Según María de la Macarena Iribarne (2010), este modelo es una deriva reactualizada del paradigma de la "maternidad científica" (Jaggar, 1988) e ideológicamente se fundamenta en tres principios básicos: una ingente inversión de tiempo, dinero y apego en la crianza, de ahí que se la vincule también con la llamada "crianza con apego" o "crianza respetuosa" (Mantilla, 2019; Sánchez de Bustamante, 2019); el ninguneo del aporte paterno a esta crianza y la exaltación de la infancia como etapa sagrada y pura por naturaleza (Visa, 2019: 283).

Si bien, tal y como señala Visa, este modelo está siendo paulatinamente desplazado por otro que no duda en mostrar la cara menos amable de la maternidad, representando las frustraciones, los cambios emocionales o las dificultades financieras que implica su experiencia en las sociedades contemporáneas, el hecho de que, a principios de mayo del año 2019, por ejemplo, la cadena El Corte Inglés publicitara el Día de la Madre en España con la fotografía de una mujer bien acicalada y sonriente, y lo acompañara con el siguiente texto: "97\% entregada 3\% egoísta $0 \%$ quejas $100 \%$ madre", pone de manifiesto la vigencia de este ejemplo de maternidad abnegada y sacrificada, cuyos deseos son interpretados como egoísmo y sus demandas como quejas. Por otro lado, evidencia también el grado de devaluación que estas dos últimas cuestiones -los deseos y las demandasadquieren en el imaginario sociocultural, lo que repercute de manera directa en las posibilidades de creación y/o aplicación de políticas públicas concretas que favorezcan las condiciones de vida de quienes maternan en el cotidiano.

El proyecto de generar un mapa federal de cuidados por parte del nuevo Ministerio de las Mujeres, Géneros y Diversidad de la nación argentina a lo largo del 2020, dentro del cual se incluirían las maternidades como trabajo de cuidados permanente, supone la promesa de un resarcimiento en este sentido. No obstante, este gesto político no se explica sin tener en cuenta las diversas propuestas de la sociedad civil que, desde hace más de una década, vienen impulsando acciones en torno a ésta y otras tantas cuestiones vinculadas a los delineamientos de la maternidad. En esta línea, cabe destacar grupos consolidados y de alcance nacional como Las Casildas o la Liga de la Leche, que han desarrollado una extensa labor tanto en lo virtual como en lo territorial en torno a cuestiones como el parto respetado, la denuncia de la violencia obstétrica o la defensa de la lactancia materna con una clara orientación de género. En paralelo, han ido proliferando una serie de propuestas de base, de alcance más bien local y con una fuerte impronta territorial, como son las rondas de gestantes, de puerperio, de apoyo a la lactancia materna o las formaciones y encuentros de doulas, en las que ciertas consignas feministas referidas a la autodeterminación sexual o la autonomía corporal se van combinando con una mirada holística y desde los derechos humanos aplicados a los procesos de la maternidad (Felitti y Abdala, 2018). A ello hay que 
sumarle, a partir del año 2017, la proyección y vitalidad virtual de una serie de grupos y páginas en las redes sociales (Facebook e Instagram, sobre todo), en los que la articulación entre maternidad y feminismo es central.

En este estudio todas estas expresiones son incluidas dentro de un movimiento mayor, con una trayectoria histórica y transnacional (Vivas, 2019: 116-118), al que en estas páginas denomino Movimiento Activista Materno (a partir de ahora MAM), por estar protagonizado, sobre todo, por madres ${ }^{2}$ que llevan a cabo su activismo a la par que maternan, poniendo en el centro de sus demandas a la maternidad. Coincido con Carolina del Olmo al juzgar que no es un movimiento uniforme ni coherente en algunas de las posturas que lo integran, pero sí que manifiesta una potencialidad crítica y emancipadora interesante al tener como objetivo principal "[...] la búsqueda de una solución a los malestares que genera la contradicción fundamental entre capitalismo y crianza" (Olmo, 2013: 68). Esta doble valencia del MAM se explica por los procesos de apropiación y uso por parte de las subjetividades, lo que favorece que muchas veces se produzcan refiguraciones en los procesos de subjetivación maternos que no necesariamente implican una ruptura con el orden establecido, pero acontecen y son capaces de generar transformaciones sin una guía garantizada (Papalini, 2013).

Un ejemplo paradigmático de esto último lo encontramos en la convocatoria del \#piquetetazo que tuvo lugar en toda la Argentina en el año 2016, después de que una mujer-madre joven que amamantaba en una plaza pública de una localidad bonaerense fuera obligada por la policía a retirarse del lugar (Magallanes y Bard Wigdor, 2017). La rápida respuesta que este hecho produjo en la sociedad refleja el crecimiento en número y visibilidad del MAM: en este caso, fueron mujeres-madres que, a través de las redes sociales, se autoconvocaron para ocupar un espacio ciudadano (las plazas) y mostrar una práctica de la intimidad (el amamantamiento) en público, en un entorno festivo de reivindicación, porteando, en cochecito, con los hijos/as en brazos, todas ellas unidas por un mismo propósito, legitimar la lactancia humana como bien social y como recurso para el empoderamiento de las madres.

La apertura de significaciones que este tipo de acciones habilitan, al ubicar la subjetividad materna en el centro de la enunciación y facilitar, así, su agenciamiento en la construcción de narrativas en torno a esta etapa vital, son fundamentales para comprender la importancia que el MAM puede llegar a tener a la hora de interrogarnos sobre los imaginarios de nuestra época en relación a la maternidad, la crianza, el cuidado, la familia y los roles de género. Por eso mismo, en este artículo se parte de la descripción y análisis de dos propuestas del MAM, con el objetivo de abordar qué tipo de retóricas son las que se ponen en juego en estas (re)construcciones subjetivas y discursivas de la vivencia materna, en especial en un contexto de grandes debates en torno a los derechos sexuales y (no) reproductivos de las mujeres y las identidades disidentes en América Latina.

\section{Metodología}

Este estudio se asienta sobre una investigación cualitativa en torno al MAM, dentro del cual se abordan los contenidos discursivos de las propuestas virtuales y territoriales que lo integran. Se parte de considerar la noción de "discurso" desde la perspectiva de la crítica ideológica (Asensi, 2011; Man, 1990; Foucault, 2006) y, en este sentido, se utiliza el Análisis Crítico del Discurso (ACD) como herramienta para explorar textualidades de diversa naturaleza semiótica (conversaciones espontáneas, comentarios en redes sociales, etc.), y analizar en ellas qué tipo de mensajes o imaginarios se presentan respecto a lo que puede ser vivible, visible y narrable de la maternidad; si estos pueden llegar a devenir desestabilizadores de ciertos órdenes simbólicos, y de qué manera.

Concretamente, para este artículo se han seleccionado dos ejemplos de un corpus mucho mayor en el que se incluyen las múltiples expresiones del MAM, anteriormente mencionadas. Los criterios que se han seguido para esta delimitación han sido temporales (2018-2019), espaciales (Córdoba), temáticos (en ambos casos, la construcción de un modelo determinado de maternidad y de subjetividad materna está intrínsecamente ligado a la concepción del embarazo, el parto y el post-parto inmediato como vivencias que dotan de identidad a quienes las atraviesan) y metodológicos. A través de un trabajo de campo móvil (Gutiérrez, 2016: 35-39), en el que se ha seguido el principio de la etnografía feminista de multisituar la investigación empírica tanto en contextos geográficos concretos como en lugares de concentración de la creatividad personal o colectiva (Castañeda, 2012: 229), se han dividido las fuentes de análisis en dos grupos: de un lado, los presenciales, dentro de los cuales se han incluido las rondas o círculos de gestantes y de puerperio, así como las formaciones y los encuentros mensuales de doulas. Del otro, los virtuales, dentro de los cuales se han tenido en cuenta organizaciones y colectivos consolidados, así como grupos en formación en redes sociales. Cabe señalar que se trata de una división estratégica, ya que las interacciones y continuidades entre uno y otro ámbito son constantes y definitorias, también, de los modos de constituirse en objetos de análisis (Hine, 2004: 78).

El hecho de que Internet ponga al alcance de las subjetividades un espacio más amplio para la expresión personal y para su realización como comunicadoras, autoras, transmisoras y receptoras de la información (La-

Refiero aquí la forma común de denominación, si bien es cierto que han proliferado en los últimos tiempos modos inclusivos, en los que se emplea la "x" para nombrar aquellas otras experiencias no identificadas con la sexualización heteronormativa. 
rrondo, 2005: 381) permite considerar la potencialidad del vínculo entre el ciberespacio y la expansión actual del MAM. En especial, si tenemos en cuenta que uno de los principales reclamos de las madres es que no son escuchadas ni tenidas en cuenta como sujetos de derecho y de deseo (Cañero Ruiz, 2019; Merino, 2017). La siguiente reflexión de la activista materna que coordina la página Des-madre en Argentina desde el año 2016 da buena cuenta de esto último:

"Los feminismos piden a gritos perspectiva sobre la transversalidad de opresiones. Me cansa leer el capacitismo que exige 'salir a la calle' para contrarrestar el prejuicio instalado, terríblemente retrógrado y conservador de que las redes virtuales son una isla o la paja de algo más grande.

Ante los medios masivos funcionales las redes son nuestra voz, el espacio contrahegemónico con el que contamos las personas que no estamos incluídas en el prime time de la televisión ni en el discurso mainstream del 'sentido común'.

Y por otra parte es la trinchera de quienes, por el motivo que sea, todo el tiempo o algunas veces, no podemos salir de nuestras casas.

Es un argumento misógino, además, en nuestro entorno, donde sabemos de qué se trata la exclusión de mujeres madres y personas cuidadoras de los espacios comunes, o que muchas veces evitamos participar en espacios políticos porque prácticamente nunca son apto niñes y el adultocentrismo normalizado, que jamás es sutil, se hace sentir" (06/02/2018).

Tal y como se infiere de estas palabras, la virtualidad no solo permite la reapropiación, creación y transmisión de sentidos por parte de las subjetividades excluidas de aquellos ámbitos considerados tradicionalmente como públicos (la calle), sino también, y muy especialmente, la redefinición de los límites que separan lo público de lo privado (Salvador, 2010). En este marco, puede ser también un buen escenario para observar cómo éstas accionan, reaccionan, construyen estrategias y posturas, ponen en práctica micropolíticas cotidianas y se entraman con esos feminismos a los que la activista interpela en la primera línea de su reflexión (García y Silva, 2017: 281).

Una de las hipótesis principales que guía esta investigación mayor en torno al MAM es que, a partir de la eclosión y popularización de los feminismos en el territorio argentino desde la primera gran marcha del $3 \mathrm{~J}$ en el año 2015, este movimiento crece y se expande en interacción continua -y no exenta de tensiones- con aquellos. A través del trabajo de campo llevado a cabo en los últimos cinco años se ha podido advertir, por un lado, el crecimiento en número y visibilidad de las subjetividades que se auto-definen como madres feministas, tanto en las redes sociales como en la cotidianeidad y variedad de sus prácticas. Y, por el otro, una mayor frecuencia de actividades compartidas entre ambos movimientos. Un buen ejemplo de ello es la inclusión de temas como la violencia obstétrica en los últimos manifiestos colectivos convocando al paro del $8 \mathrm{M}$ de la agrupación argentina \#NiUnaMenos (2017, punto 5; y 2018, punto 6) o la participación conjunta en espacios no solo ciudadanos, como en el anteriormente mencionado caso del \#piquetetazo, sino también, y sobre todo, feministas. Destaco, en este último sentido, las intervenciones bajo la misma bandera del grupo de Facebook Crianzas feministas Cba durante la última marcha por el 8M (2019); de la Colectiva Maternidades Feministas en el marco del último Encuentro Nacional de Mujeres 2019 (a partir de esa fecha, Encuentro Plurinacional y Disidente); o el aporte de Mujeres por un Parto Respetado Córdoba en el I Simposio de Arte, Política y Feminismo: Hacia nuevas imágenes, narraciones y sentidos en relación con el aborto, que tuvo lugar en la ciudad de Córdoba en agosto del 2019.

Tal y como ya fuera adelantado en el apartado anterior, las investigaciones feministas han aportado diversas lecturas en torno a la maternidad. Después de varias décadas de trabajo crítico para separarla de la construcción identitaria de la sujeto mujer, se observará de qué manera estas propuestas se relacionan con esta premisa fundamental del feminismo y con el movimiento en general, qué (re)figuraciones las lleva a generar y desde qué perspectiva lo hacen. Si la maternidad, como constructo cultural, puede ser analizada desde el conjunto de efectos producidos por el despliegue de diversas tecnologías, entre ellas las de género (Sánchez de Bustamante, 2019), abordar el lenguaje de las mujeres en espacios que son considerados propios y apropiados, permite reflexionar en torno a las contradicciones, tensiones y fisuras que sus narrativas sostienen o abren respecto a esta identidad generizada. También en torno a las disputas de sentido que se producen en los procesos socioculturales cuando las subjetividades pugnan por "[hacer] vivible la inequidad, o bien colarse por las rendijas para plantear cambios y alternativas ante las pretensiones hegemónicas de recrear y reproducir la desigualdad de todo signo" (Castañeda, 2012: 235).

\section{Análisis}

En los apartados que siguen se analizan, en primer lugar, los encuentros mensuales Nosotras Doulas. Espacio de profundización y aprendizaje, acontecidos en una localidad cercana de Córdoba capital durante los años 2017, 2018 y parte del 2019. Los mismos son iniciativa de una partera y una doula, coordinadoras del proyecto Nacer para amar, dentro del cual se efectúan, también, rondas quincenales para parejas gestantes (a las que 
nombran como Círculo gestante familiar) y una Formación de doulas anual (con una periodicidad mensual). El único requisito para participar del espacio de encuentro es haber sido formado/a ${ }^{3}$ como doula (aunque no se ejerza), ya sea en esta formación, ya sea en alguna de las que existen a nivel nacional e internacional. En segundo lugar, se abordan algunos de los textos que la colectiva Mujeres por un Parto Respetado Córdoba (a partir de ahora MPRC) puso en circulación en el marco de distintas campañas activistas.

Es importante señalar que este tipo de propuestas se inscriben en un lugar intermedio entre los movimientos de salud de las mujeres que, en las décadas de los sesenta y setenta del siglo XX, lucharon por el reconocimiento de las mujeres como sujetas activas, con capacidad para tomar decisiones respecto a cuestiones concernientes a su salud sexual y (no) reproductiva, y con el derecho a recibir información completa y actualizada sobre esta área (Felitti, 2011b). Y la emergencia contemporánea del feminismo como movimiento de denuncia de las desigualdades y elementos de poder incluidos en el lenguaje y naturalizados en las prácticas cotidianas (Lagarde, 1996). El resultado de ocupar este lugar de frontera es, como ya se dijo, la intervención cada vez más frecuente de muchas de estas iniciativas en espacios de lucha feminista. Pero también la producción de narrativas en las que ciertos imaginarios de maternidad se entremezclan con el uso de recursos lingüísticos inclusivos, por ejemplo, provocando así continuidades y desplazamientos en la circulación de sentidos en torno al rol materno como experiencia generizada y generizante.

\subsection{Los encuentros de doulas: la maternidad encarnada}

Se pregunta Carolina del Olmo (2013) dónde quedan nuestras tribus en estas sociedades post-industriales individualistas y arrasadas por un neoliberalismo descarnado. Las familias se han ido acortando a una o dos personas adultas, a lo sumo, tres (los/as abuelos/as), que participan externamente de algunos asuntos de la crianza -"ayudando" o "apoyando", más que ejerciendo. Asimismo, las redes de sostenes que se tejían alrededor de las mujeres en generaciones no tan lejanas se han perdido. Y, sin embargo, las exigencias de la maternidad se hacen cada vez más complejas e inasumibles, por cuanto obligan a muchas madres a escindirse entre sus deseos de realización personal y laboral, y el amor a los/as hijos/as (Tadey, 2015).

En este contexto, la figura de la doula emerge como una subjetividad compleja e interesante. Los procesos de globalización actuales, caracterizados por el flujo de personas e ideas, y la creación de redes virtuales, puede explicar la importación de esta figura desde el norte global. Por otro lado, la extensión de un paradigma de atención a la salud fundado en criterios de patologización y medicalización indefinida (Foucault, 1976) justificaría su aceptación entre las capas medias y altas de sociedades como la argentina, donde la violencia obstétrica sigue siendo uno de los principales problemas que enfrentan las mujeres y personas gestantes en el ámbito del sistema sanitario (Canevari, 2011).

Según las definiciones más extendidas de su rol, las doulas son acompañantes emocionales de otras mujeres en los procesos de gestación, parto y puerperio. Eventualmente pueden acompañar pérdidas gestacionales, principalmente involuntarias (el llamado duelo perinatal), aunque cada vez más, también, voluntarias. En el caso de estas últimas, convocadas más por la amistad-que por el servicio en sí. La ilegalidad del aborto en Argentina, así como el hecho de que, hasta hace muy poco, éste haya sido un tema tabú entre las mujeres, explica que no haya una orientación específica en este tipo de acompañamientos, como sí puede haberla en el caso del puerperio, por ejemplo. Los cambios producidos en la sociedad argentina, con momentos bisagra como el debate sobre el aborto en el congreso (de marzo a agosto de 2018) o la denuncia pública por violación contra Juan Darthés del Colectivo Actrices Argentinas (en diciembre de 2018), a través de los cuales se dieron a conocer miles de testimonios similares en las redes y en las conversaciones cotidianas, evidencian la urgente necesidad de una parte importante de la población de visibilizar(se), de contar(se) y de entramar(se) (en) una red de apoyo que trascienda sus vínculos inmediatos. Una circunstancia, ésta, de la que no son ajenos espacios como el que aquí analizo, donde la cuestión del aborto generó un interesante debate entre quienes lo leían en clave de derechos y de autonomía decisional, y quienes sostenían imaginarios conservadores y naturalistas, de corte más bien liberal, en los que se hablaba de una incoherencia entre la acción de tener relaciones sexuales y la decisión de abortar (Nota de campo 1). Una conversación posterior, sostenida en el grupo de whatsapp del grupo de encuentros, puso de manifiesto que el prejuicio de una de las personas que defendía esta última postura estaba más atravesado por una experiencia personal no resuelta (un aborto clandestino vivido con culpa y necesidad de negación) que por tener una opinión crítica al respecto.

Suele ser frecuente el dicho de que cualquier mujer que haya acompañado a otra en su camino a la maternidad desde el amor, el cuidado y el respeto ha manifestado su ser doula, evidenciando así la añoranza por un pasado de unión y solidaridad en el que la maternidad ocuparía un lugar central en la conformación de una organización social de género en femenino. No obstante, la proliferación de formaciones en Córdoba y en otras provincias de Argentina es un claro exponente de que esta subjetividad sigue en construcción. Las hay que admiten entre sus alumnas a personas que no han sido madres, otras que proponen este criterio como excluyente.

Utilizo ambos marcadores morfológicos porque a partir de la segunda mitad del año 2018 participó un varón heterosexual, quien también se estaba formando con las coordinadoras del espacio. 
Incluso algunas admiten a varones cis entre sus alumnas, en un gesto experimental que nace de un deseo tanto de integrar a ese "otro" masculino a un proceso que se considera esencialmente femenino, como de ser parte en la aceptación e integración de su lado más femenino. En este sentido, de acuerdo con Felitti y Abdala (2018) estas propuestas deben ser leídas en interacción constante con la reactualizada cultura de la Nueva Era, pero siempre teniendo en cuenta que, en lo que se refiere a las subjetividades, las modulaciones de las que ellas son capaces muchas veces extralimitan y redefinen los conceptos que las sujetan a modos de hacer y de ser. Solo a modo de ejemplo, en el último encuentro del 2018 la presencia del hombre arrastró una discusión en torno al uso real y simbólico del lenguaje. Se discutió la posibilidad de nombrarlo como doulo, y se reflexionó acerca de la pertenencia e implicancias de usar el lenguaje inclusivo o los nuevos modismos feminizadores (hablar de "cuerpa" o de "útera", por ejemplo). Después de un breve intercambio, se llegó a la conclusión de que nombrarlo como doula era la mejor opción, no solo por ser un término extranjero de difícil traducción, sino como una forma de asegurar un orden de género femenino predominante, pero no excluyente (Nota de campo 8).

Por otro lado, su dedicación al campo psicoafectivo de la mujer y, en menor medida, de la pareja y la familia, les otorga un papel interesante en los procesos socioculturales de reconfiguración de imaginarios maternos. En el caso analizado, la asistencia mayoritariamente de mujeres-madres cis, formadas como doulas, permite considerar una serie de puntos en común. Para empezar, todas ellas comparten experiencias a la hora de formarse y de acercarse posteriormente a un espacio que se define como "[...] un círculo sagrado y diverso entre Doulas. Es una red de sostén, respeto, hermandad, compromiso. Es un cuenco de unión, reflexión y discernimiento, que nutre nuestro preciado rol, y desde aquí nos visibilizamos mas [sic] claras": muchas han atravesado episodios de violencia médica institucional (la violencia obstétrica) en alguno de sus embarazos y partos, así como en su relación con la lactancia y con la crianza en general; otras han parido en sus casas en compañía de una partera y de una doula u, ocasionalmente, de una mujer cumpliendo un rol similar; la mayoría han amamantado durante períodos prolongados; casi todas han porteado a o colechan con sus hijos/as, a quienes suelen llevar a escuelas alternativas y a los/as que no vacunan. Las hay, incluso, que viven este acercamiento como una forma de prepararse a conciencia para sus futuras maternidades y quienes se lo toman, más bien, como parte de un camino de realización personal en el que buscan sanar alguna cuestión de su ser hija, de su ser mujer o de su ser madre. Esto genera un vaivén constante entre las dimensiones personales de cada una, sus búsquedas, anhelos y necesidades, y el salto hacia una dimensión más colectiva, que no solo las hace sentirse parte de una red de mujeres sino también, y muy especialmente, animarse a seguir reproduciendo la misma lógica aprendida por medio de la creación y apertura de círculos varios de acompañamiento ${ }^{4}$.

En este escenario, se perfila una concepción bastante homogénea de maternidad, en la que la subjetivación de este tipo de prácticas es la base para la construcción identitaria y generizada de la experiencia materna. Los libros de la bióloga española Casilda Rodrigáñez (2009; en co-autoría con Cachafeiro, 2007) son una referencia clave en este tipo de propuestas, en las cuales se refleja también una corriente del feminismo espiritual en la que la celebración de los procesos reproductivos (entre los que se encuentra la maternidad) apunta a la apertura de significaciones - más positivas- en torno a los mismos. Como han demostrado los estudios entorno a las nuevas corrientes de espiritualidad femenina (Ramírez, 2014 y 2019), "el eje está puesto precisamente en las relaciones entre género y poder [...], en definiciones de empoderamiento que también apuntan a lo introspectivo en tanto se promociona el conocimiento y disfrute del propio cuerpo, a partir de la celebración de procesos sexuales y reproductivos" (Felitti, 2019: 153).

De acuerdo con esta mirada, las mujeres en su experiencia de maternidad adquieren un papel protagónico en la construcción de una nueva humanidad menos violenta y más respetuosa con el entorno (humano y ambiental). La famosa frase, repetida de manera acrítica en sucesivos encuentros, marchas y rondas: "Para cambiar el mundo es necesario cambiar la forma de nacer", del obstetra francés pionero del movimiento del parto sin dolor, Michel Odent (2011), vendría a resumir esta idea, a la que se suma también la voluntad de recuperar aquellos saberes y haceres que se consideran olvidados, negados o expropiados por el poder médico masculino, y masculinizado en sus prácticas. A ello refiere la consigna "El parto siempre será nuestro", que dos de las mujeres que asisten a estos encuentros eligieron en el marco de la convocatoria para el 8M 2019 de Crianzas feministas Cba. La misma consistió en cambiar los perfiles personales de Facebook con una fotografía y una frase que resumiera qué entendía cada integrante de este grupo por criar en clave feminista.

Esta última cuestión sobre la reapropiación de saberes y haceres es lo que, también, justificaría prácticas como las anteriormente mencionadas, donde todo el peso recae en los vínculos corporales, de la mujer con su (nuevo) cuerpo materno y de este con el cuerpo del/a bebé. Esta centralidad otorgada a la materialidad del cuerpo como espacio para vincularse con el cuerpo del/a otro/a (específicamente, del/a bebé), y que ha sido duramente cuestionada por un sector del feminismo contemporáneo por esencialista, esclavizante y desigual (Badinter, 2011; Bobel, 2001; Gimeno, 2018) es reivindicado en este tipo de espacios como eje central en los

\footnotetext{
Así, por ejemplo, en los últimos meses del 2018 y primeros del 2019 se abrieron tres círculos de puerperio dentro del grupo de doulas que participan del Nosotras Doulas. Los tres estaban dedicados al puerperio inicial (de 0 a 9 meses), eran coordinados por dos mujeres doulas y se congregaban en distintos lugares de la provincia (uno en un barrio de Córdoba Capital y los otros dos en localidades cercanas). Paralelamente a estas ofertas, surgieron otras propuestas cercanas pero con temáticas más concretas, como son las "ceremonias prenatales", también conocidas como "bendiciones de camino" o "despedidas de panzas", o los talleres de fertilidad para parejas, todos ellos coordinados por una dupla de mujeres doulas.
} 
procesos de autonomización y construcción subjetiva materna. Ello quedó especialmente claro cuando, en el último encuentro del 2018 en el que una de las coordinadoras del espacio compartió una serie de consideraciones acerca del cuidado corporal durante el post-parto, al proponer nombrar su enfoque como "encuerpado" y referir a las consideraciones de Claudia Korol al respecto (2016: 15), mi aporte fue recibido con especial efusividad por las personas presentes y fue muy agradecido por la otra coordinadora, quien manifestó sentirse plenamente definida por el término (Nota de campo 8). Esta cuestión fue relevante también para la reconsideración del vínculo con el feminismo como movimiento con el que se sienten en constante tensión: de saberse poco representadas o cuestionadas por posturas como las mencionadas más arriba, pasaron a encontrar una agenda en común con aquellas otras miradas, más populares y comunitarias, que reclaman por el cuerpo como primer territorio soberano ante las múltiples formas de expresión de la violencia patriarcal, y que reivindican la sanación (personal y colectiva) como camino político (Cabnal, 2010).

\subsection{Mujeres por un Parto Respetado Córdoba: activismos emancipatorios}

\section{Señalaba hace unos años Patricia Merino en su libro Maternidad, Igualdad y Fraternidad. Las madres como} sujeto político en las sociedades poslaborales:

"una parte del feminismo coincide con la visión hegemónica de que gestar, parir, amamantar y criar son actividades que no merecen ser objeto de atención por parte de la ciudadanía, hechos sociales a los que no conviene dar una centralidad política, y en cualquier caso, no dotan a quien los realiza de identidad" (2017: 431; el subrayado es suyo).

Dos años después de estas reflexiones, Esther Vivas destacaba la importancia de "valorar [estas prácticas], visibilizarlas pública y políticamente y revindicar la responsabilidad colectiva en el marco de un proyecto social emancipador" (2019: 115).

En este marco, cabe destacar la labor de la colectiva MPRC, una agrupación de carácter local que viene trabajando en la politización de la gestación y el parto desde una perspectiva de derechos que implica, hoy, hablar de una maternidad deseada y elegida. El hecho de que en los últimos años gran parte de sus actividades en la red se hayan concentrado en el acompañamiento y visibilización de la lucha ciudadana por el aborto legal, seguro y gratuito, dando continuidad a sus acciones en torno a la defensa del parto respetado y la denuncia de la violencia obstétrica como violencia de género, esboza una línea de pensamiento compartido con los reclamos feministas en torno al derecho a la autodeterminación sexual y corporal de mujeres y disidencias, así como con las movilizaciones contra las violencias cotidianas que sufren estos colectivos.

Actualmente, MPRC está conformada por nueve mujeres, y su objetivo principal es difundir información y concientizar a la población en general sobre los derechos jurídicos que benefician particularmente a mujeres -pero también, desde hace apenas unos pocos años, a "lesbianas, travestis y trans" (MPRC, 21/11/2018)- a partir de la reglamentación de la ley 25.929 (Ley de Parto Respetado o Ley de Parto Humanizado) y la sanción de las leyes 26.484 (Ley Nacional e Integral para Prevenir, Sancionar y Erradicar la Violencia contra las Mujeres) y 26.529 (Ley de Derechos del Paciente). Esto es lo que las lleva a crear campañas como "Por el derecho a elegir dónde, cómo y con quién parir", en la que se invitó a participar a aquellas familias que quisieran posicionarse en contra del Proyecto de Ley 20-70-D-20185 con la publicación de fotografías de sus hijos/as (y el texto "Yo nací en casa acompañado/a por una partera") o de su intimidad (y el texto "Yo/Nosotros/as parimos en casa acompañada/os/as por una partera"). También a recordar en cada uno de los cinco afiches de la campaña "Violencia obstétrica es violencia de género" la siguiente consigna: "Ejerzamos nuestros derechos. Tenemos leyes: 25.929 y 26.485". Y a acompañar la descripción de la campaña "Menos intervenciones, más cuidados" con el siguiente pedido: "[...] que en hospitales, maternidades, clínicas y domicilios se garanticen los derechos de la ley 25.929 de madres, padres e hijxs en el proceso de nacimiento" (MPRC, 14/05/2018).

Junto a estas, MPRC ha realizado un total de ocho campañas de difusión, visibilización y sensibilización respecto a dos puntos: por un lado, el cuestionamiento al sistema biomédico que considera el parto como un evento patológico, que concibe el cuerpo como una máquina y al/a paciente como un objeto fragmentado, y que jerarquiza y estandariza los cuidados con vistas, muchas veces, a un beneficio económico (Felitti y Abdala, 2018). Y, por el otro, la construcción de un modelo alternativo de atención basado en el respeto a la autonomía decisional de las sujetos gestantes; a su protagonismo en el momento del embarazo, parto y postparto; y, finalmente, a sus posibilidades emancipatorias a partir del agenciamiento del saber del cuerpo. En términos generales, todas sus acciones se han orientado a la denuncia de la violencia obstétrica como violencia estructural de género, y a la defensa del parto respetado como derecho sexual y reproductivo. Asimismo, en la realidad física, coordinan charlas-debates, realizan exposiciones fotográficas en instituciones públicas y de

El Proyecto de Ley 20-70-D-2018 fue presentado en el año 2018 por el Partido para una República con Oportunidades (PRO), dirigido por el entonces presidente de la Argentina Mauricio Macri, a los fines de delimitar los alcances del ejercicio de la obstetricia. De acuerdo a este proyecto, solo los y las profesionales recibidos/as como obstetras podrían acompañar el embarazo, el parto y el post-parto, lo que excluiría de la legalidad a las parteras, doulas y puericultoras, quienes actualmente cubren las necesidades de quienes deciden recibir un acompañamiento fuera del marco institucional. Esto limitaría también la posibilidad de decidir y elegir cómo, dónde y con quién parir de las mujeres y las personas gestantes, así como de sus familias. 
acceso abierto como hospitales y maternidades, universidades o centros culturales, y llevan a cabo talleres para personas gestantes y para profesionales en los centros de salud de los barrios.

Esta doble labor, tanto en el ámbito de lo virtual como en el de lo territorial, la ha convertido en una de las principales referentes del Movimiento por un Parto Humanizado en la provincia de Córdoba, lo que le otorga un valor social y público fundamental. De hecho, a lo largo del trabajo de campo se ha podido constatar cómo gran parte de las campañas o comunicaciones publicadas en su página de Facebook son inmediatamente replicadas en los perfiles de muchas de las personas que participan de los encuentros de doulas, quienes, además, mantienen vínculos personales y profesionales con algunas de las integrantes de la colectiva. Esto permite observar el juego de continuidades y desplazamientos de sentidos que se dan entre dos propuestas de temática similar, pero con enfoques y propósitos diversos: en este caso concreto de la colectiva, las configuraciones de la maternidad están íntimamente ligadas a la mostración -fotográfica y narrativa- del embarazo, el parto y el post-parto inmediato como procesos biológicos y fisiológicos ineludibles para la subjetivación materna. Pero es precisamente esta exposición pública de algo que se considera del ámbito de lo doméstico y de lo íntimo lo que les permite trascender ciertas lecturas apolíticas de la maternidad y de los procesos a ella asociados, tal y como es denunciado por Patricia Merino o Esther Vivas en sus respectivos libros; y, a su vez, tensar las limitaciones de considerarla única y exclusivamente del orden de lo biológico y no como una responsabilidad colectiva.

Esta última cuestión es la que ha impulsado campañas como la "Violencia obstétrica es violencia de género", destinada a "desnaturalizar las prácticas violentas" y, de manera específica, "detener prácticas que deterioran y lastiman el tejido social de nuestra comunidad" (MPRC, 21/11/2018). No es difícil descubrir la huella del pensamiento de la antropóloga feminista argentina Rita Segato resonando en esta última frase. Suya es la idea de que "[h]ay que hacer la política del día a día, por fuera del Estado: retejer el tejido comunitario, derrumbar los muros que encapsulan los espacios domésticos y restaurar la politicidad de lo doméstico propia de la vida comunal" (2016: 106; el subrayado es de mi autoría). A ello apuntan, pues, campañas como ésta o "Menos intervenciones, más cuidados", en la que se pueden distinguir, también, y siguiendo el hilo de la lectura propuesta por Segato, "[...] una retórica de valor, un vocabulario de defensa al camino relacional, a las formas de felicidad comunales, que pueda contraponerse a la poderosa retórica del proyecto de las cosas, meritocrático, productivista, desarrollista y concentrador" (2016: 106).

A continuación propongo la lectura de una de las narrativas puestas en circulación en este marco, para concluir con el análisis. Se trata de un texto publicado durante la campaña "Violencia obstétrica es violencia de género", que consistió en la difusión de cinco afiches, todos ellos con una representación similar: junto al título de la campaña, se incluía la información sobre los derechos referidos a la temática y se explicitaba la denuncia a cuatro prácticas consideradas naturalizadas en su estandarización institucional: "Nos inmovilizan para parir", "Cortan nuestros genitales de rutina (episiotomía)", "Presionan nuestra panza y empujan nuestrxs bebés (maniobra de Kristeller)" y "Nos separan de nuestrxs bebés". A su vez, cada afiche contenía, de fondo, un retazo de fotografía con algún detalle hospitalario: el costado de una incubadora, las patas de una camilla, una tijera y un crucifijo arriba de un fragmento del instrumental médico. De esta forma se lograba generar un campo figurativo alrededor de los sucesivos cuestionamientos: porque no solo se trataba de lo que el afiche expresaba a través de las palabras y de la imagen, sino de las narrativas que los acompañaban en el apartado correspondiente de la red social Facebook destinada a la escritura. Destaco el que acompañó el último afiche de la campaña, "Nos separan de nuestrxs bebés":

"El inmediato contacto piel a piel tras el nacimiento es crucial para la vida de lxs recién nacidxs y su [sic] madres. Desde el inicio del vínculo, ambos se reconocen y se estabiliza la respiración y oxigenación del bebé y sus niveles de glucemia. Se estabiliza también la presión arterial, se reducen las hormonas del estrés, disminuye el llanto, se incrementa el estado de alerta tranquila y se promueve el inicio precoz de la lactancia materna. Además, se mantiene la temperatura estable, lo cual reduce los riesgos de hipotermia. Quien acompaña puede observar signos vitales (color, respiración, mirada, respuesta motriz) en el pecho de la madre. La separación precoz del recién nacidx de su madre, el cortado inmediato del cordón umbilical, los controles rutinarios y sin urgencia fuera de sus brazos, están desaconsejados por la OMS por sus consecuencias negativas. La separación violenta los nacimientos" (MPRC, 26/11/2018).

Haciendo uso de las recomendaciones que la OMS nombrara en la Declaración de Fortaleza de 1985 y ampliando el alcance de su crítica a factores físicos, psíquicos y sexuales de la vida y la salud de las mujeres y de los/as bebés, la colectiva encuadraba dentro de las múltiples formas de manifestación de la violencia obstétrica una práctica médica institucional muy común, como es la separación prematura entre el cuerpo materno y el cuerpo del/a bebé, y la contraponía a un modelo de atención que respetaría las necesidades bio-emocionales de ambos. Al poner en el centro de su planteamiento la interdependencia como característica fundamental de la experiencia materna y, por extensión, de la vida humana, la colectiva se hacía eco de una de las cuestiones centrales en la agenda feminista latinoamericana: el cuidado de la vida en todas sus formas y expresiones como principio no solo antipatriarcal, sino también anticapitalista. Por otro lado, al utilizar la herramienta retórica de la contraposición de haceres y miradas, favorecía que el foco se desplazara de objetivo: de la maternidad 
propiamente dicha, con todas las obturaciones que su discurso esencializante puede llegar a reproducir sin una vigilancia epistémica contante, a un sistema violento y disciplinador de los cuerpos, de los sentires y de las emociones.

\section{Conclusiones}

"La maternidad será deseada, o no será". Esta frase que se repite en contextos distintos y que sigue articulando las corrientes feministas en América Latina, no solo nos interpela a seguir luchando por los derechos (no) reproductivos de una parte importante de la población, sino a explorar qué tipo de deseo es el que dignifica las experiencias y prácticas de maternidad en las sociedades neoliberales del presente.

En este artículo me he propuesto describir y analizar dos ejemplos de lo que he dado en llamar el Movimiento Activista Materno, y cuyo objetivo general es disputar un modelo de maternidad único y hegemónico a partir de la producción y reproducción de relatos y prácticas en torno a la experiencia de maternidad. Por medio de actividades diversas en la realidad física y virtual, estas propuestas ponen de manifiesto la importancia de comprender esta etapa de la vida de una persona desde una perspectiva abierta y plural, multideterminada por un sinfin de factores, entre ellos, el de género.

En función de esto, se escogieron dos iniciativas de la sociedad civil: de un lado, los encuentros territoriales de doulas que se vienen sucediendo desde hace tres años en una localidad cercana a la ciudad argentina de Córdoba; y, del otro, algunas de las campañas virtuales que la colectiva Mujeres por un Parto Respetado Córdoba ha realizado en los últimos años. Se advirtió la riqueza de matices que abre esta doble pertenencia a un movimiento que bebe de las luchas por el derecho a la salud sexual y (no) reproductiva de las mujeres durante la segunda mitad del siglo XX y a otro que reivindica la maternidad como responsabilidad social y a las madres como sujetos políticos (Merino, 2017).

Se observó que ambas se nutren de conceptos provenientes de una nueva espiritualidad que ancla en el cuerpo la recuperación de saberes y haceres en clave femenina, al mismo tiempo que reivindica sus procesos biológicos como potencialidad transformadora.

Es precisamente este énfasis puesto en la materialidad del cuerpo lo que genera algunas de las discusiones más interesantes alrededor de sus narrativas, ya que, en definitiva, el cuerpo que se sigue legitimando en estos contextos es un organismo binariamente sexuado y fisiológicamente verdadero. Esto provoca la obturación en modelos de maternidad bastante homogéneos y excluyentes de otras tantas experiencias, como las que hoy en día aportan los colectivos de lesbianas, personas trans, no binarias, queer o de género fluido que optan por la adopción o la reproducción asistida para acceder a la maternidad, e, incluso, las mujeres cis no heterosexuales que no comulgan con esta subjetivación corporal de su identidad materna. No obstante, considero que la politización de algunos de estos procesos vinculados al imaginario biológico de la maternidad, así como su mostración en contextos de comunicación masiva y cambiante de sentido como es el universo de internet y de las redes sociales, habilita una apertura de sentidos respecto a las posibilidades de comprender este esencialismo desde la perspectiva estratégica anunciada por Spivak (1993).

No en vano, la reevaluación de la maternidad y de su materialidad corporal en estos discursos está muy ligada a la circulación de ideas en torno a la autonomía sexual, la soberanía corporal o la militancia por los derechos de las mujeres. Si bien es cierto que, de los ejemplos analizados, MPRC parece evidenciar con más claridad esta amalgama, a veces contradictoria, entre posicionamientos y demandas, no es menor el hecho de que sus campañas sean replicadas y acompañadas por muchas de las mujeres que participan de los encuentros de doulas.

\section{Referencias bibliográficas}

Asensi, Manuel (2011). Crítica y sabotaje. Barcelona: Anthropos.

Badinter, Élisabeth (2011). La mujer y la madre. Un libro polémico sobre la maternidad como nueva forma de esclavitud. Madrid: La Esfera de los Libros.

Beauvoir, Simone de (2005). El segundo sexo. Madrid: Cátedra.

Bobel, Chris (2001). Bounded Liberation: A Focused Study of La Leche League International. Gender and Society, 15 (1), $130-$ 151. doi: 10.1177/089124301015001007

Cabnal, Lorena. 2010. Acercamiento a la construcción de la propuesta de pensamiento epistémico de las mujeres indígenas feministas comunitarias de Abya Yala. Disponible en: www.acsur.org. (Consultado el 04/05/2018).

Canevari, Cecilia. (2011). Cuerpos enajenados: experiencias de mujeres en una universidad pública. Santiago del Estero: FHCSyS-UNSE/Barco Edita.

Cañero, Julia. (2019). Crítica al antimaternalismo. Píkara magazine. Recuperado de https://bit.ly/2urJuSq.

Castañeda, Martha Patricia (2012). Etnografía feminista. En Norma Blázquez, Fátima Flores y Maribel Ríos (comps): Investigación feminista. Epistemología, metodología y representaciones sociales (pp. 217-238). México: UNAM /Centro de Investigaciones Interdisciplinarias en Ciencias y Humanidades: Centro Regional de Investigaciones Multidisciplinarias: Facultad de Psicología. 
Chodorow, Nancy (1984). El ejercicio de la maternidad. Psicoanálisis y sociología de la maternidad y paternidad en la crianza de los hijos. Barcelona: Gedisa.

Des-Madre (06/02/2018). Los feminismos piden a gritos. Disponible en: https://bit.ly/32m2DSA (consultado el 07/02/2018).

Federici, Silvia (2013). Revolución en punto cero. Trabajo doméstico, reproducción y luchas feministas. Madrid: Traficantes de sueños.

Federici, Silvia (2015). Calibán y la bruja. Mujeres, cuerpo y acumulación originaria. Buenos Aires: Tinta Limón.

Felitti, Karina (2011a). Introducción. En Karina Felitti (comp.): Madre no hay una sola. Experiencias de maternidad en la Argentina (pp. 11-21). CABA: Ciccus Ediciones.

Felitti, Karina. (2011b). Parirás sin dolor: poder médico, género y política en las nuevas formas de atención del parto en la Argentina (1960-1980), Histórica, Ciências, Saúde-Manguinhos, 18 (supl. 1), 113-129.

Felitti, Karina (2019). Brujas de la Nueva Era. La salud de las mujeres en clave espiritual y feminista. En: Fundación Soberanía Sanitaria (comp.): Salud feminista. Soberanía de los cuerpos, poder y organización (pp. 147-160). CABA: Tinta Limón.

Felitti, Karina y Abdala, Leila (2018). El parto humanizado en la Argentina: activismos, espiritualidades y derechos. En Georgina Sánchez Ramírez y Hanna Laako (eds.): Parterías de Latinoamérica. Diferentes territorios, mismas batallas (pp. 95-121). San Cristóbal de las Casas /Chiapas/México: El Colegio de la Frontera Sur.

Foucault, Michel (1976). La crisis de la medicina o la crisis de la antimedicina, Educación médica y salud, 2, 152-169. Disponible en: https://bit.ly/37YWr3X (consultado el 30/10/2017).

Foucault, Michel (2006). Sobre la ilustración. Madrid: Tecnos.

García, Almudena y Silva e Silva, Artenira. (2017). Ciberfeminismo o feminismo en la red: Haciendo arqueología en internet. Antropología Experimental, 17, 277-286.

Gimeno, Beatriz. (2018). La lactancia materna. Política e identidad. Madrid: Cátedra.

Gutiérrez, Ana Paulina (2016). Etnografía móvil: una posibilidad metodológica para el análisis de las identidades de género en Facebook, Revista Interdisciplinaria de Estudios de Género, 4, 26-45.

Hays, Sharon (1998). Las contradicciones culturales de la maternidad. Barcelona: Paidós.

Iribarne, María de la Macarena (2010). Discursos sobre la maternidad científica. Una perspectiva crítica, Investigaciones Feministas, 1, 193-212.

Hine, Christine (2004). Etnografia virtual. Barcelona: UOC.

Jaggar, Alisson (1988). Feminist Politics and Human Nature. Nueva Jersey: Rowman \& Littlefield Publishers.

Korol, Claudia (2016). Feminismos populares. Las brujas necesarias en los tiempos de cólera. En Claudia Korol (comp.): Feminismos populares. Pedagogías y politicas (pp. 15-25). CABA: Chirimbote.

Lagarde, Marcela (1996). Género y feminismo. Desarrollo humano y democracia. Madrid: horas y horas.

Larrondo, Ainara. (2005). La Red al servicio de las mujeres. Aproximación a la relación mujer y medios de comunicación en Internet. Estudios sobre el Mensaje Periodístico, 11, 375-392.

Magallanes, Mariana Loreta y Bard Wigdor, Gabriela (2017). \#Piquetetazo: La lucha ciberfeminista y la agenda mediática, Gênero \& Direito, 6 (3), 196-221.

Man, Paul (1990). Alegorias de la lectura. Barcelona: Lumen.

Mantilla, María Jimena (2019). Cuerpos, niñez y crianza: cartografías corporales de la infancia en el modelo de crianza respetuosa en Argentina, Revista Uruguaya de Antropología y Etnografia, 1, 61-75. doi:10.29112/RUAE.v4.n1.4

Merino, Patricia. (2017). Maternidad, Igualdad y Fraternidad. Las madres como sujeto politico en las sociedades poslaborales. Madrid: Clave Intelectual.

Mujeres por un Parto Respetado Córdoba (2019, agosto). Nuestro cuerpo, nuestro territorio. Por un paradigma del respeto. I Simposio de Arte, Politica y Feminismo, Córdoba, Universidad Nacional de Córdoba, Córdoba.

\#NiUnaMenos (8 de marzo de 2017). Manifiesto Ejes para el acto $8 \mathrm{M}$ ¿Por qué paramos?. Recuperado de https://bit.ly/33toV3M \#NiUnaMenos (9 de marzo de 2018). Manifiesto 8M2018. Recuperado de https://bit.ly/35EWgKw.

Odent, Michel (2011). El bebé es un mamífero. Tenerife: Ob Stare.

Olmo, Carolina del (2013). ¿Dónde está mi tribu? Maternidad y crianza en una sociedad individualista. CABA: Capital Intelectual.

Palomar, Cristina (2005). Maternidad: Historia y cultura. La Ventana, 22, 35-67.

Papalini, Vanina. (2013). “Tecnologías del yo": entre la gubernamentalidad y la autonomía. En Raúl Rodríguez Freire (ed.): El gobierno del presente. Materiales criticos (pp.1-18). Valparaíso: Instituto de Literatura y Ciencias del Lenguaje, Pontificia Universidad Católica de Valparaíso.

Quinzio, Patrice di (1999). The Impossibility of Motherhood: Feminism, Individualism and the Problem of Mothering. Nueva York: Routledge.

Ramírez, María del Rosario (2014). La espiritualidad femenina desde los círculos de mujeres”. En: Afef Hagi y Giovanna Campani (eds.): Conflitti social e religione nel mediterraneo (pp. 157-164). Firenze: Mauro Pagliai Editore.

Ramírez, María del Rosario (2019). Espiritualidades femeninas: el caso de los círculos de mujeres, Encartes, 3, $144-162$.

Rich, Adrienne (2019). Nacemos de mujer. La maternidad como experiencia e institución. Madrid: Traficantes de Sueños.

Rodrigáñez, Casilda (2009). Pariremos con placer. Murcia: Ediciones Crimentales.

Rodrigáñez, Casilda y Cachafeiro, Ana (2007). La represion del deseo materno y la génesis del estado de sumisión inconsciente. Murcia: Ediciones Crimentales.

Saletti, Lorena (2008). Propuestas teóricas feministas en relación al concepto de maternidad, Clepsydra, 7, 169-183.

Salvador, Saleta de. (2010). Ciberactivismo ecofeminista. Investigaciones feministas, 1, 27-41.

Sánchez de Bustamante, Marina (2019). Debates sobre crianza con apego en medios de comunicación argentinos: mamis famosas y la secta del colecho, Comunicación y Medios, 40, 14-25. doi:10.5354/0719-1529.2019.53885.

Sau, Victoria (2004). El vacio de la maternidad. Madre no hay más que ninguna. Barcelona: Icaria / Antrazyt.

Segato, Rita (2016). La guerra contra las mujeres. Madrid: Traficantes de Sueños. 
Spivak, Gayatri (1993). In a Word: Interview. En Gayatri Chakravorty Spivak: Outside in the Thinking Machine (pp. 1-23). London: Routledge.

Tadey, Mariana (2015). El dilema de las mujeres que investigan. ¿Publicar o procrear? Desde la Patagonia difundiendo saberes, 12 (20), 20-24.

Vasallo, Brigitte (12/02/2014). Desocupar la maternidad. Disponible en: https://bit.ly/2HR0Co3 (consultado el 07/02/2018).

Visa, Mariona (2019). Relatos sobre maternidad, reproducción y crianza en la era post-televisión, Investigaciones Feministas, 10 (2), 281-294. doi:https://dx.doi.org/10.5209/infe.66494

Vivas, Ester. (2019). Mamá desobediente. Una Mirada feminista a la maternidad. Madrid: Capitán Swing Libros.

\section{Fuentes digitales}

Mujeres por un Parto Respetado Córdoba (14/05/2018). Menos intervenciones, más cuidados. Disponible en: https://bit. ly/38X4nEe (consultado el 25 de marzo de 2019).

Mujeres por un Parto Respetado Córdoba (29/06/2018). NO al Proyecto de Ley 20-70-D-2018. Disponible en: https://bit. ly/2PmEjdZ (consultado el 30 de marzo de 2019).

Mujeres por un parto respetado Córdoba (26/11/2018). Nos separan de nuestrxs bebés. Disponible en: https://bit.ly/2SYeAuR (consultado el 25 de marzo de 2019).

Mujeres por un Parto Respetado Córdoba (21/11/2018). Desnaturalicemos las prácticas violentas. Disponible en: https://bit. ly/38W1Gp4 (consultado el 25 de marzo de 2019).

\section{Registros etnográficos}

Notas de campo 1, "Dejar fluir la vida. Sanando los duelos. Acompañamiento a madres, transitando el puerperio, con la experiencia de la pérdida de su bebé. Como dar voz al duelo dentro del sistema de creencias en nuestra cultura" dictado por la Lic. Mariana Herrera, Nosotras Doulas, Córdoba, mayo de 2018. Registro de AUTORA.

Notas de campo 8, "Cuidados esenciales luego del nacimiento", dictado por la partera María Eugenia Alba, Nosotras Doulas, Córdoba, diciembre de 2018. Registro de AUTORA. 